\section{Variación estacional de las defunciones por infarto agudo del miocardio en Chile}

\author{
JAIME CERDA $^{1}$, CLAUDIA BAMBS ${ }^{1}$
}

\section{Seasonal variation of acute myocardial infarction deaths in Chile}

Background: Hospitalization and deaths due to cardiovascular diseases (CVD), have a peak in frequency during winter. Aim: To assess the existence of seasonal variation in deaths due to acute myocardial infarction (AMI) in Chile. Material and Methods: Analysis of death report databases available at the website of the Chilean Ministry of Health. The seasonality of deaths due to AMI (codes ICD-10 I21, I22, I23) occuring in Chile between 2001-2016 were analyzed using a geometric model assuming a sinusoidal cyclic pattern. Results: During the period 2001-2016, a total of 94,788 deaths due to AMI were registered, corresponding to 93,349 corrected deaths. Of the latter, 29.2\% occurred in winter, $24.9 \%$ in spring, $24.0 \%$ in autumn and $21.8 \%$ in summer. The geometric model showed a marked sinusoidal pattern for the aggregated data. The peak-to-low ratio of deaths was 1.41 (95\% CI 1.38-1.44). The peak of deaths occurred during July in 14 out of 16 years analyzed. Conclusions: In Chile, deaths due to AMI have a marked seasonal pattern, characterized by a higher number of deaths in winter and a lower number in summer.

(Rev Med Chile 2021; 149: 665-671)

Key words: Myocardial Infarction; Mortality; Seasons.

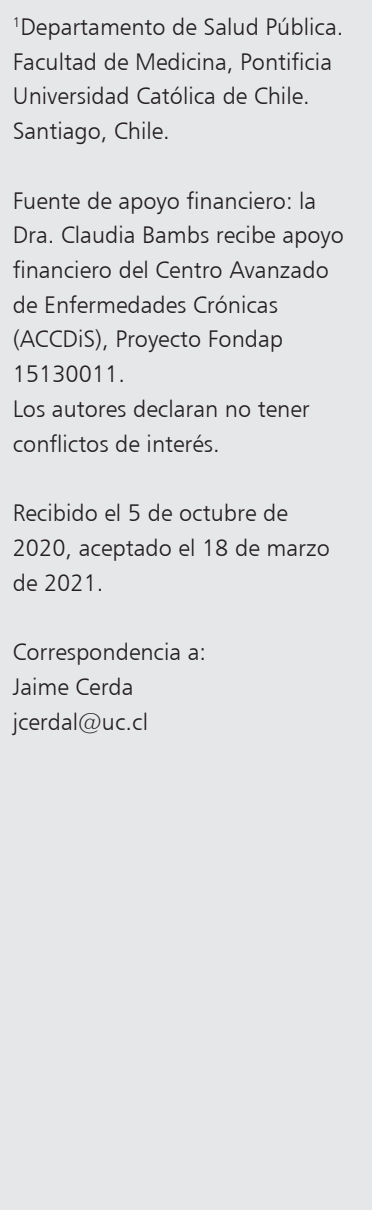

'Departamento de Salud Pública. Facultad de Medicina, Pontificia Universidad Católica de Chile.

Fuente de apoyo financiero: I Dra Claudia Bambs recibe apoyo de Enfermedades Crónicas (ACCiS), Proyecto Fondap Los autores declaran no tener conflictos de interés.

Recibido el 5 de octubre de 2020, aceptado el 18 de marzo de 2021

Correspondencia a:

Cerd

jerdal@uc.c
L a estacionalidad en la incidencia de enfermedades, definida como la ocurrencia cíclica y sistemática de estas en un período de tiempo, es un fenómeno descrito desde tiempos de Hipócrates ${ }^{1}$. En el ámbito de las enfermedades cardiovasculares (ECV), una revisión narrativa de la literatura realizada por Stewart concluyó que (i) variaciones estacionales en un amplio rango de poblaciones y climas (predominantemente europeos y en menor medida norteamericanos) han sido descrita para todos los tipos de ECV; (ii) la mayoría de los estudios reporta alzas invernales en hospitalizaciones y muertes relacionadas a ECV, siendo las tasas en invierno típicamente $20-30 \%$ mayores en comparación al nadir veraniego; $y$ (iii) objetivar este fenómeno en diferentes lugares del planeta es problemático, existiendo inconsisten- cias secundarias a la amplia heterogeneidad de métodos utilizados y discrepancias derivadas de la compleja interacción entre la susceptibilidad individual, ya sea fisiológica como conductual, y un rango de factores ambientales. Pese a la evidencia de efectividad de intervenciones para prevenir o mitigar este efecto, Stewart ${ }^{2}$ destaca que existe escasa implementación de ellas en la práctica.

En Chile, más allá de observaciones no sistematizadas, la variación estacional de las ECV ha sido escasamente investigada. Marti-Soler ${ }^{3}$ analizó la variación estacional de la mortalidad por ECV en 19 países de diferentes latitudes, pero sin especificar diagnósticos ni cuantificar el exceso de defunciones. Por su parte, Nazzal ${ }^{4}$ analizó la variación estacional de las hospitalizaciones por infarto agudo del miocardio en Chile. Ambos 
estudios demostraron la existencia de un patrón estacional, con un predominio de ocurrencia de eventos durante el invierno.

El presente estudio amplía temporalmente el análisis de Marti-Soler ${ }^{3}$ y se centra específicamente en la caracterización de la variación estacional de las defunciones por infarto agudo del miocardio (IAM) en Chile.

\section{Material y Método}

\section{Fuentes de información}

Para cada año del período 2001-2016 se obtuvo el número de defunciones por IAM registradas en Chile, correspondientes a los códigos CIE-10 "infarto agudo del miocardio" (I21), "infarto subsecuente del miocardio" (I22) y "ciertas complicaciones presentes posteriores al infarto agudo del miocardio" (I23) (Anexo 1), a partir de las bases de datos de mortalidad confeccionadas por el Departamento de Estadísticas e Información en Salud (DEIS). Esta información es de libre acceso, existiendo una base de datos por año. Anualmente, las defunciones registradas por IAM fueron estratificadas según mes del año y corregidas por la duración del mes, según la fórmula [defunciones corregidas $=($ defunciones observadas $* 30) /$ días del mes)]. Estas fueron denominadas defunciones por IAM corregidas, $y$ fueron utilizadas para efecto de cálculos y análisis.

\section{Análisis estadístico}

Las defunciones por IAM corregidas fueron reagrupadas en las cuatro estaciones meteorológicas del hemisferio sur: verano (diciembre, enero y febrero), otoño (marzo, abril y mayo), invierno (junio, julio y agosto) y primavera (septiembre, octubre y noviembre). Este ordenamiento generó una serie de tiempo compuesta por 64 datos (4 estaciones meteorológicas ${ }^{\star} 16$ años). Se calculó la proporción de defunciones por IAM correspondiente a cada estación meteorológica, en base a los datos agregados del período 2001-2016.

Con el propósito de diferenciar el componente estacional y el componente de tendencia en la serie de tiempo, se realizó un modelo de regresión lineal simple, correspondiendo el residuo de cada dato de la serie de tiempo al componente estacional. Para cada estación meteorológica se estimó el valor promedio de los residuos obtenidos durante el período 2001-2016 en cada año, permitiendo de esta forma caracterizar el componente estacional libre del componente de tendencia (i.e. detrended).

Se usó una segunda forma de analizar estacionalidad a través de un modelo geométrico asumiendo un patrón cíclico sinusoidal ${ }^{5}$. Esta estrategia se basó en una regresión armónica (periódica) realizada para cada año y para los datos agregados del período en estudio. Para ello, se utilizó la hoja de cálculo Episheet ${ }^{6}$, de libre acceso. Episheet entregó una representación gráfica de la variación estacional, una estimación de la fecha en que se produce el número máximo de defunciones por IAM (day of peak) y la razón máximo/mínimo (peak-to-low ratio) de defunciones por IAM con su respectivo intervalo de confianza 95\%. Para efectuar las estimaciones, la única información requerida por la hoja de cálculo fue el número de defunciones por IAM de cada mes del año analizado.

Por tratarse del análisis de una base de datos de acceso público, el presente estudio fue eximido de revisión ética por la Unidad de Ética y Seguridad de la Pontificia Universidad Católica de Chile.

\section{Resultados}

En Chile, durante el período 2001-2016 se registró un total de 94.788 defunciones por IAM (códigos CIE-10 I21, I22, I23). El número de defunciones por IAM presentó un valor mínimo el año 2009 (5.466 defunciones) y máximo el año 2007 (6.415 defunciones) (Tabla 1). Por su parte, el número total de defunciones por IAM corregidas para el período en estudio fue 93.349. De ellas, $27.292(29,2 \%)$ ocurrieron en invierno; 23.282 $(24,9 \%)$ en primavera; $20.378(21,8 \%)$ en verano y $22.397(24,0 \%)$ en otoño. La Figura 1 ilustra la variación estacional de las defunciones por IAM durante el período 2001-2016. Con excepción del último año, en todos los años el número máximo ocurrió en invierno y el mínimo en verano.

El análisis de tendencia de la serie de tiempo realizado mediante regresión lineal simple presentó una tendencia de $+1,695$ defunciones por IAM por cada estación-año analizada. El promedio de los residuos obtenido para cada estación fue +246 (invierno), -6 (primavera), -182 (verano) y -58 (otoño), confirmando la presencia de un patrón estacional, esta vez habiendo eliminado el componente de tendencia. 
Tabla 1. Defunciones por infarto agudo del miocardio, registradas y corregidas

(Chile, 2001-2016)

\begin{tabular}{|ccc|}
\hline Año (b) & $\begin{array}{c}\text { Defunciones por infarto agudo } \\
\text { del miocardio (a) }\end{array}$ \\
\hline 2001 & 5.684 & 5.601 \\
Registradas & Corregidas \\
\hline 2002 & 5.801 & 5.719 \\
\hline 2003 & 6.055 & 5.963 \\
\hline 2004 & 5.754 & 5.657 \\
\hline 2005 & 5.834 & 5.753 \\
\hline 2006 & 5.853 & 5.765 \\
\hline 2007 & 6.415 & 6.320 \\
\hline 2008 & 5.719 & 5.622 \\
\hline 2009 & 5.466 & 5.384 \\
\hline 2010 & 5.964 & 5.880 \\
\hline 2011 & 5.599 & 5.516 \\
\hline 2012 & 6.049 & 5.945 \\
\hline 2013 & 6.029 & 5.940 \\
\hline 2014 & 6.062 & 5.976 \\
\hline 2015 & 6.280 & 6.188 \\
\hline 2016 & 6.224 & 6.120 \\
\hline Todos los años & 94.788 & 93.349 \\
\hline
\end{tabular}

(a) Códigos ClE-10: 121, 122, 123. (b)Cada año incluye el mes de diciembre del año anterior, y excluye el mes de diciembre del año en curso.
Tanto para la totalidad del período en estudio como para cada año en particular, las defunciones por IAM siguieron un patrón sinusoidal, siendo adecuadamente ajustadas por el modelo geométrico (Figura 2). La razón máximo/mínimo (peak-to-low ratio) para cada año del período en estudio fluctuó entre 1,31 y 1,56, siendo en todos los años estadísticamente significativa. Agrupando la totalidad de defunciones por IAM del período en estudio, dicha razón presentó un valor de 1,41 (IC95\% 1,38-1,44). Por su parte, la estimación de la fecha en que ocurrió el número máximo de defunciones por IAM (day of peak) para cada año del período en estudio fluctuó entre el 1 de julio y el 16 de agosto; en 14 de los 16 años estudiados el punto máximo se registró en el mes de julio y en 2 de los 16 años estudiados en el mes de agosto. Agrupando la totalidad de defunciones por IAM del período en estudio, el modelo estimó la ocurrencia del valor máximo de defunciones por IAM el día 21 de julio (Tabla 2).

\section{Discusión}

El presente estudio demuestra la existencia de un patrón estacional en la mortalidad por IAM en Chile durante el período 2001-2016. Dicha estacionalidad se caracteriza por un número mayor de defunciones ocurridas en invierno, en comparación con verano.

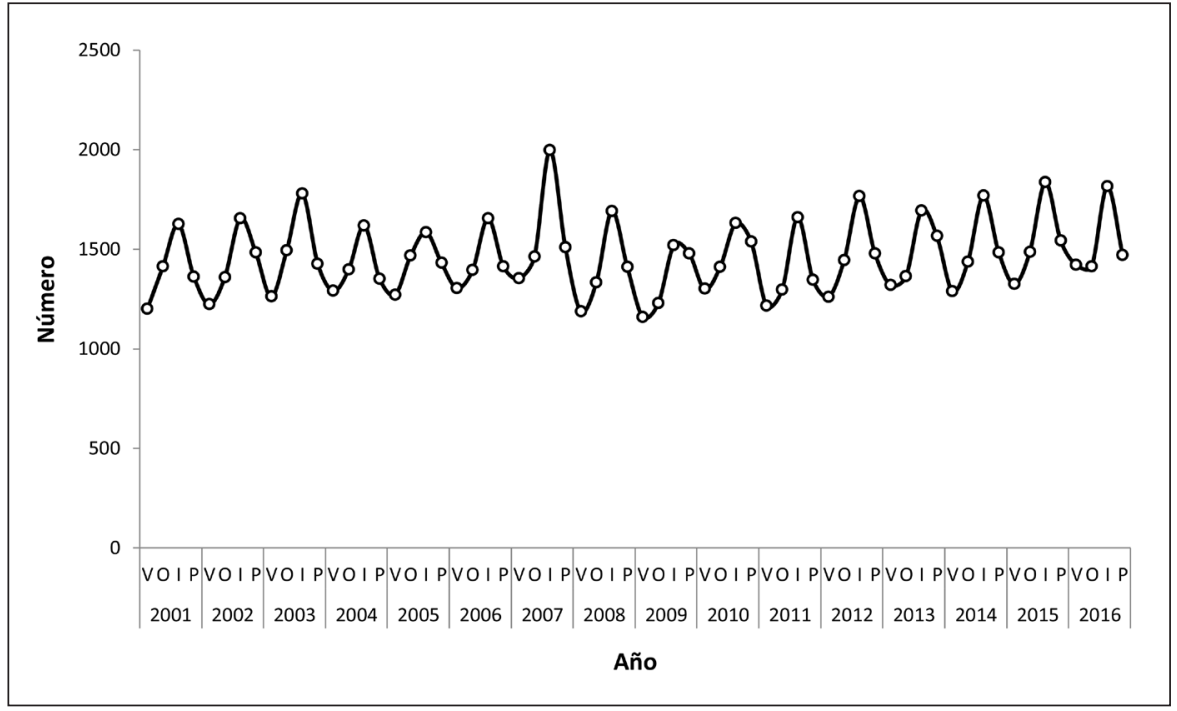

Figura 1. Defunciones por infarto agudo al miocardio, según estación (Chile, 2001-2016). V: verano, O: otoño, I: invierno, P: primavera. 


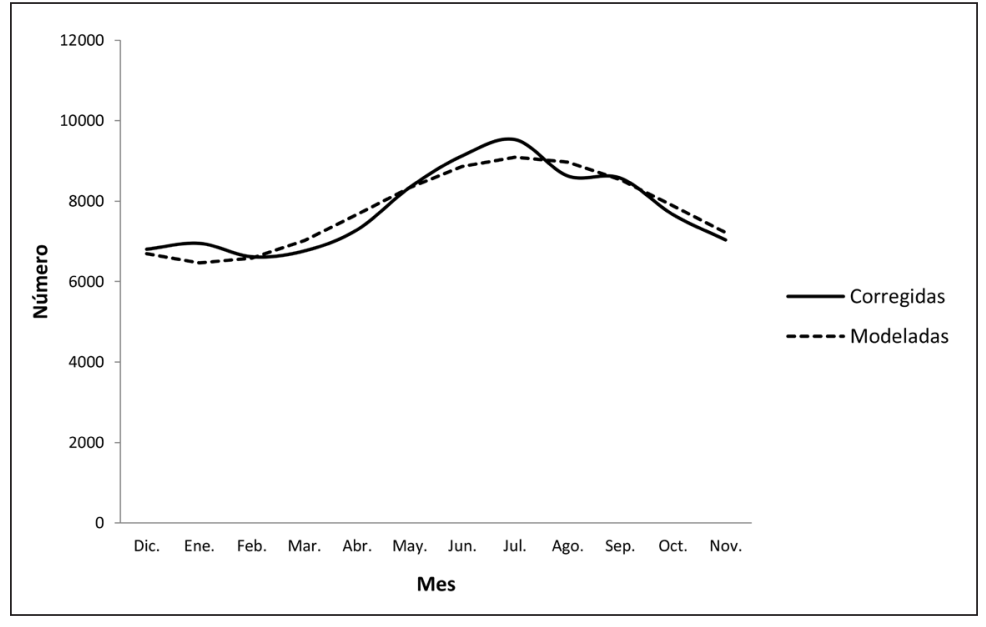

Figura 2. Defunciones por infarto agudo al miocardio, corregidas y modeladas, según mes del año (Chile, 2001-2016).

Tabla 2. Estimación de la razón de defunciones por infarto agudo del miocardio (a) máximo/mínimo, fecha del día máximo y su estación meteorológica (Chile, 2001-2016)

\begin{tabular}{|cccc|}
\hline Año (b) & $\begin{array}{c}\text { Razón máximo/mínimo } \\
\text { (peak-to-Iow ratio) }\end{array}$ & $\begin{array}{c}\text { Fecha del día máximo } \\
\text { (day of peak) }\end{array}$ & $\begin{array}{c}\text { Estación } \\
\text { meteorológica }\end{array}$ \\
\hline 2001 & 1,44 & Jul. 10 & Invierno \\
\hline 2002 & 1,46 & Jul. 23 & Invierno \\
\hline 2003 & 1,47 & Jul. 15 & Invierno \\
\hline 2004 & 1,31 & Jul. 09 & Invierno \\
\hline 2005 & 1,35 & Jul. 01 & Invierno \\
\hline 2006 & 1,31 & Jul. 23 & Invierno \\
\hline 2007 & 1,56 & Jul. 17 & Invierno \\
\hline 2008 & 1,45 & Jul. 25 & Invierno \\
\hline 2009 & 1,44 & Ago. 16 & Invierno \\
\hline 2010 & 1,34 & Jul. 26 & Invierno \\
\hline 2011 & 1,43 & Jul. 24 & Invierno \\
\hline 2012 & 1,46 & Jul. 20 & Invierno \\
\hline 2013 & 1,42 & Ago. 09 & Invierno \\
\hline 2014 & 1,38 & Jul. 14 & Invierno \\
\hline 2015 & 1,42 & Jul. 22 & Invierno \\
\hline 2016 & 1,36 & Jul. 17 & Invierno \\
\hline Todos los años & 1,41 & Jul. 21 & \\
\hline
\end{tabular}

(a) Códigos CIE-10: 121, 122, 123. Los casos analizados corresponden a defunciones corregidas. (b) Cada año incluye el mes de diciembre del año anterior, y excluye el mes de diciembre del año en curso.

En Chile, en 15 de los 16 años del período en estudio, el valor máximo y mínimo de defunciones ocurrió en la estación meteorológica de invierno y verano, respectivamente. El predominio de defunciones por IAM durante los meses de invierno concuerda con el exceso de mortalidad por ECV reportado por Marti-Soler ${ }^{3}$ en 19 países ubicados en distintas latitudes (incluido Chile), así como con el estudio chileno de base poblacional de hospitalizaciones por IAM realizado por $\mathrm{Nazzal}^{4}$. La evidencia acumulada sugiere que en Chile, tanto la incidencia de IAM (reflejada en las hospitali- 
zaciones por este diagnóstico), como la letalidad del mismo siguen un comportamiento estacional común, siendo el invierno la estación de mayor riesgo. Ello permite plantear que las condiciones presentes e intensificadas en esta estación podrían precipitar la ocurrencia de un IAM, así como favorecer un desenlace adverso.

La variación estacional de las ECV refleja una compleja interacción entre la susceptibilidad individual, tanto fisiológica como conductual, y un amplio rango de factores ambientales, destacando principalmente la temperatura y la contaminación ambiental. Con respecto a la temperatura ambiental, se han descrito alzas de morbilidad y mortalidad por ECV tanto en invierno como en verano, varios de ellos asociados a olas de frío y de calor; de igual forma, las transiciones y variabilidad de temperatura también pueden gatillar la ocurrencia de un IAM. Por su parte, la revisión sistemática realizada por Mustafic ${ }^{7}$ concluyó que los principales contaminantes ambientales (monóxido de carbono, dióxido de nitrógeno, dióxido de sulfuro, así como material particulado $\mathrm{PM}_{2.5}$ y $\mathrm{PM}_{10}$ ) se asocian de manera significativa a un aumento en el riesgo de IAM a corto plazo. Lo anterior concuerda con el hecho de que la temporada invernal es la que presenta históricamente los peores índices de calidad del aire en Chile. Es importante destacar que tanto para la temperatura ambiental como para la contaminación ambiental se han descrito potenciales mecanismos fisiopatológicos que explicarían su vinculación con la ocurrencia de IAM, reforzando la plausibilidad de una posible relación de tipo causa/efecto. Por ejemplo, los potenciales mecanismos que explican un mayor riesgo de eventos coronarios luego de la exposición al frío actuarían por medio de tres vías: (a) Estimulación de receptores cutáneos $\rightarrow$ aumento de niveles de catecolaminas $\rightarrow$ vasoconstricción y aumento de frecuencia cardíaca y presión arterial $\rightarrow$ isquemia miocárdica e inestabilidad de placa coronaria; (b) Aumento de la diuresis $\rightarrow$ disminución de volumen plasmático y hemoconcentración $\rightarrow$ aumento de la viscosidad sanguínea $\rightarrow$ aumento de la concentración plasmática de factores de coagulación y recuento plaquetario $\rightarrow$ trombosis; y (c) Inhalación de aire frío $\rightarrow$ reflejos pulmonares neurogénicos $\rightarrow$ potenciación de vulnerabilidad a aterotrombosis y arritmia ${ }^{8}$.

Una de las consecuencias del cambio climático planetario será la ocurrencia de eventos climáticos extremos más frecuentes, con el potencial de afectar negativamente la salud cardiovascular, especialmente en personas susceptibles, debido a su limitada capacidad de adaptación ${ }^{8}$. Un grupo de especial interés son los adultos mayores. Destaca que entre los años 2002 y 2016, el índice de adultos mayores en Chile, calculado según (población $60+$ años/población 0-14 años)*100, aumentó de $40,9 \%$ a $75,9 \%$, reflejando el acelerado proceso de envejecimiento de nuestra población ${ }^{9}$, aumentando el número de personas susceptibles. A ello se agrega una alta carga de factores de riesgo cardiovascular y deficiencia de vitamina $\mathrm{D}$ presente en la población chilena ${ }^{10}$, altos niveles de contaminación ambiental y circulación de virus respiratorios documentada cada invierno en Santiago y otras ciudades del país. Con respecto a este último punto, Kwong reportó una asociación significativa entre infecciones respiratorias (especialmente influenza) y hospitalizaciones por IAM, siendo la razón de incidencia de dicho desenlace en los 7 días posteriores a la constatación de un test positivo para influenza (i.e. período de riesgo) seis veces mayor comparado con el año previo y el año posterior (i.e. período de control) a la constatación de la infección ${ }^{11}$.

La demostración de un patrón estacional en las defunciones por IAM tiene implicancias en términos preventivos. Al respecto, se ha propuesto un conjunto de estrategias individuales y poblacionales, las cuales tendrían el potencial de reducir la mortalidad por IAM, patología cuya letalidad ha sido estimada en aproximadamente $50 \%{ }^{12}$. Dentro de las estrategias de base individual, enfocadas en sujetos de alto riesgo, se encuentran evitar el estrés térmico mediante uso de calefacción/aire acondicionado, restricción de actividad física al aire libre en áreas contaminadas (especialmente en personas de mayor riesgo cardiovascular), vacunación anual antiinfluenza y antineumocócica del adulto, suplementación de vitamina $\mathrm{D}$ y tratamiento del trastorno afectivo estacional; a esto se agrega la educación de la ciudadanía en autocuidado, así como la difusión amplia de reportes de condiciones de temperatura y contaminación ambiental actualizados, veraces y comprensibles. Por su parte, las estrategias de tipo poblacional corresponden a políticas de subsidio de climatización de hogares, sistemas de alerta de eventos climáticos extremos (olas de frío y calor) y estrategias de mitigación 
de contaminación ambiental ${ }^{2,8}$. Desde un punto de vista de gestión, nuestros resultados abren la discusión sobre la eventual necesidad de reforzar durante el invierno las unidades de cuidados intensivos que reciben a pacientes coronarios a lo largo de nuestro país.

Por último, el presente estudio plantea una serie de desafíos metodológicos a resolver por futuras investigaciones epidemiológicas. Por ejemplo, evaluar si la variación estacional presenta diferencias por subgrupos determinados por el sexo, edad, localización geográfica o nivel socioeconómico, entre otras variables. Asimismo, es de interés caracterizar a los sujetos que fallecen por IAM durante el invierno y verano, con el fin de evaluar el peso real de factores individuales y estacionales sobre la incidencia y letalidad de esta patología. En la medida que se cuente con esta información, los profesionales de la salud y tomadores de decisiones podrán implementar estrategias preventivas centradas principalmente en los grupos más susceptibles. Por otro lado, Skajaa ${ }^{13}$ advierte la posible ocurrencia de sesgo en la estimación de la razón máximo/mínimo (peakto-low ratio), consistente en que parte de su valor puede atribuirse al azar. Para explorar la magnitud de dicho sesgo en nuestros datos realizamos un ejercicio de simulación, en el cual distribuimos de manera aleatoria en los 12 meses del año las defunciones mensuales por IAM de todo el período. Este procedimiento se repitió 100 veces, calculándose cada vez la razón máximo/mínimo (peak-to-low ratio). El promedio de dicha razón fue 1,14 (valor mínimo $=1,01$; valor máximo $=1,32$ ) valor que pudiese considerarse como un estimador del componente de la razón máximo/mínimo atribuible al azar. Tomando en cuenta que el valor mínimo que puede tomar la razón máximo/mínimo es 1,00 , al descontar la razón promedio obtenida vía simulación de la razón observada, es decir $(1,41$ $1,14)+1=1,27$, consideramos el resultado aún apoya la existencia de un componente estacional en la mortalidad por IAM.

En conclusión, el presente estudio demuestra que en Chile las defunciones por IAM presentan un patrón estacional, concentrándose en invierno. Este hallazgo puede contribuir a reforzar acciones preventivas sobre los factores de riesgo modificables que se presentan e intensifican durante esta estación.

\section{Anexo 1. Código CIE-10 incluidos bajo la definición de "infarto agudo del miocardio".}

\section{I21 Infarto agudo del miocardio}

121.0 Infarto transmural agudo del miocardio de la pared anterior

121.1 Infarto transmural agudo del miocardio de la pared inferior

121.2 Infarto agudo transmural del miocardio de otros sitios

121.3 Infarto transmural agudo del miocardio, de sitio no especificado

121.4 Infarto subendocárdico agudo del miocardio

121.9 Infarto agudo del miocardio, sin otra especificación

I22 Infarto subsecuente del miocardio

122.0 Infarto subsecuente del miocardio de la pared anterior

122.1 Infarto subsecuente del miocardio de la pared inferior

122.8 Infarto subsecuente del miocardio de otros sitios

122.9 Infarto subsecuente del miocardio, de parte no especificada
I23 Ciertas complicaciones presentes posteriores al infarto agudo del miocardio

123.0 Hemopericardio como complicación presente posterior al infarto agudo del miocardio

I23.1 Defecto del tabique auricular como complicación presente posterior al infarto del miocardio

123.2 Defecto del tabique ventricular como complicación presente posterior al infarto del miocardio

123.3 Ruptura de la pared cardíaca sin hemopericardio como complicación presente posterior al infarto agudo del miocardio

123.4 Ruptura de las cuerdas tendinosas como complicación presente posterior al infarto agudo del miocardio

123.5 Ruptura de músculo papilar como complicación presente posterior al infarto agudo del miocardio

123.6 Trombosis de la aurícula, apéndice auricular y ventrículo como complicación presente posterior al infarto agudo del miocardio

I23.8 Otras complicaciones presentes posteriores al infarto agudo del miocardio 


\section{Referencias}

1. Naumova E. Mystery if seasonality: getting the rhythm of nature. J Public Health Policy 2006; 27: 2-12.

2. Stewart S, Keates AK, Redfern A, McMurray JJV. Seasonal variations in cardiovascular disease. Nat Rev Cardiol 2017; 14: 654-64.

3. Marti-Soler H, Gonseth S, Gubelmann C, Stringhini S, Bovet P, Chen PC, et al. Seasonal Variation of Overall and Cardiovascular Mortality: A Study in 19 Countries from Different Geographic Locations. PLoS ONE 2014; 9: e113500.

4. Nazzal C, Alonso F. Variación estacional de las hospitalizaciones por infarto agudo al miocardio según sexo y edad en Chile. Rev Med Chile 2018; 146: 1233-40.

5. Christiansen CF, Pedersen L, Sørensen HT, Rothman KJ. Methods to assess seasonal effects in epidemiological studies of infectious diseases - exemplified by application to the occurrence of meningococcal disease. Clin Microbiol Infect 2012; 18: 963-9.

6. Rothman KJ. Episheet. Disponible en: http://krothman. org (Accedido el 27/07/2020).

7. Mustafic H, Jabre P, Caussin C, Murad MH, Escolano $\mathrm{S}$, Tafflet $\mathrm{M}$, et al. Main air pollutants and myocardial infarction: a systematic review and meta-analysis. JAMA 2012; 307: 713-21.

8. Claeys M, Rajagopalan S, Nawrot T, Brook R. Climate and environmental triggers of acute myocardial infarction. Eur Heart J 2017; 38: 955-60.

9. Instituto Nacional de Estadísticas. Proyecciones de Población. Disponible en: http://www.ine.cl (Accedido el 27/07/2020).

10. Margozzini P, Passi A. Encuesta Nacional de Salud, ENS 2016-2017: un aporte a la planificación sanitaria y políticas públicas en Chile. Ars Medica 2018; 43: 30-4.

11. Kwong JC, Schwartz KL, Campitelli MA, Chung H, Crowcroft NS, Karnauchow T, et al. Acute Myocardial Infarction after Laboratory-Confirmed Influenza Infection. N Engl J Med 2018; 378: 345-53.

12. Nazzal C, Alonso F. Incidencia y letalidad por infarto agudo del miocardio en Chile: 2001-2007. Rev Med Chile 2011; 139: 1253-60.

13. Skaaja N, Horváth-Puhó E, Sundbøll J, Adelborg K, Rothman KJ, Sørensen HT. Forty-year Seasonality Trends in Occurrence of Myocardial Infarction, Ischemic Stroke, and Hemorrhagic Stroke. Epidemiology 2018; 29: $777-83$. 